\title{
ALAIN BIENAYMÉ
}

Université Paris Dauphine, LEDA

\section{La nature de la firme à l'ère du numérique}

Les entreprises développent depuis vingt ans une double relation avec l'univers numérique. Les unes en conçoivent les outils, toutes les utilisent plus ou moins intensément. La fibre optique et le dispositif wifi innervent tous les secteurs de la société. Cette révolution transforme la nature de la firme. L'information se voit reconnaître un rôle stratégique. Le management développe jusqu'à la démesure deux propriétés des TIC : leur omniprésence et l'ambition de tout quantifier. La transition numérique, révolution d'un nouveau genre, entraîne de nombreux effets sur l'appareil de production du pays. 
$\mathrm{L}$ es entreprises développent depuis vingt ans une double relation avec l'outil numérique. Les unes conçoivent les matériels, les logiciels et les applications, toutes en font un usage plus ou moins intensif. La fibre optique et le dispositif wifi innervent tous les secteurs de la société, jusqu'au domicile des particuliers. Aujourd'hui l'information multilatérale se propage à la vitesse de la lumière. Comment dès lors conserver intacte la vision de la firme que Ronald Coase exposa en 1937, à l'apogée du tube pneumatique et du téléphone à cadran ? Sa conception de la nature de la firme décrit en effet une pyramide hiérarchique dirigée du sommet par un nombre encore limité d'abonnés aux PTT. En quoi l'économie numérique impose-t-elle de revoir d'un œil neuf cet acteur essentiel de l'économie de marché ? En fait, l'apport de Coase s'inscrit dans une longue évolution au terme de laquelle l'information s'est vu reconnaître un statut de matière première d'un nouveau genre. Cette étape aide à prendre la mesure des changements que l'emploi systématique des TIC apporte aujourd'hui dans les pratiques managériales comme dans les structures de l'organisation industrielle.

\section{I - LA PLACE DE L'INFORMATION DANS LA THÉORIE DE L'ENTREPRISE}

Au $19^{\circ}$ siècle les économistes se contentèrent d'assimiler l'entreprise à un lieu de production baignant dans l'océan des marchés, un point sans dimension situé à l'intersection du marché des biens et de ceux du capital et du travail. Ni l'information, ni la production manufacturière ne suscitait l'intérêt. D'une part, les marchés étaient supposés dispenser une information exhaustive sur les prix et la qualité des biens. D'autre part, les concurrents étaient censés pouvoir s'approprier gratuitement les meilleurs procédés de fabrication du moment. Dans ce monde sans inégalités de compétences ni envie, la logique d'entreprise se distingue le moins possible de celle de l'échange marchand : l'entrepreneur consacre son activité à échanger des quantités de facteurs contre des quantités de biens dans des proportions qui dépendent essentiellement de leurs prix respectifs et accessoirement d'une contrainte technique exogène. Les particularités propres à la production ne sont, hormis la loi des rendements décroissants, aucunement prises en compte. L'entreprise bénéficie comme tous les acteurs du marché d'une information égale pour tous. Ce paradigme de l'économie du tout-marché a perduré pendant des décennies. Dans les années 1960, un auteur de renom, K. Boulding, pouvait encore affirmer dans son manuel que l'échange expliquait à lui seul $90 \%$ de l'activité économique.

Or, c'est méconnaître le vrai rôle de l'information que d'en postuler la gratuité et la disponibilité sans restriction. Les marchés concrets possèdent chacun leur mystère ; celui de l'immobilier ne ressemble pas à celui des smartphones. Et, à plus forte raison, considérer que l'entreprise est une organisation parfaitement transparente relève de l'hypothèse d'école, trompeuse pour beaucoup : les entreprises ont, sinon leur jardin secret, une vie intérieure qui leur reste propre, ne serait-ce que parce que leurs dirigeants tiennent à filtrer les informations au mieux de leurs intérêts stratégiques. 
Trois auteurs ont remis en cause la vision originelle de la firme, sans toutefois concéder explicitement à l'information un rôle central. A. Marshall avance, le premier, le concept de «business organisation» appliqué à gérer des processus de production qui impliquent des relations de travail distinctes de l'échange marchand (1890). F. Knight voit de son côté dans l'entreprise une entité confrontée à un univers de risques inassurables à son échelle (1921). Devant ce défi, R. Coase (1937) met en jeu le concept d'économie des coûts de transaction. Selon lui, l'entreprise trouve son avantage à signer des contrats liant durablement l'employeur à son personnel. En effet ce procédé libère l'employeur de l'obligation de recruter sa main-d'œuvre au jour le jour. Il assure une relative sécurité d'emploi pour le salarié. En contrepartie, ce type de contrats établit une hiérarchie; il rémunère les employés au temps de travail plutôt qu'à la tâche, ce qui permet de contrôler à moindre frais le sérieux du travail accompli à travers la qualité des produits. Soit autant de traits qui préservent l'organisation de l'opportunisme des individus et des aléas du marché. Sans évoquer explicitement l'information, ces analyses ont le mérite de souligner la nature spécifique de la firme qui devient un objet scientifique clairement distinct du marché. Tant que la loi des rendements décroissants du travail et du capital limitait la taille des entreprises et qu'elles étaient censées se spécialiser dans un seul bien, il leur suffisait pour survivre, en théorie, d'ajuster la quantité produite à celle qui égalise le coût minimum de production au prix de marché de concurrence pure et parfaite. Les manuels d'économie ont insisté sur cette tâche simple où le décideur se borne à calculer le coût moyen et le coût marginal correspondant à son volume de production. Un modèle aussi rudimentaire et au contenu aussi pauvre en information ne pouvait rendre compte de la croissance des entreprises au moment où la deuxième révolution industrielle révélait l'importance du progrès techniques, des économies d'échelle liées à la fabrication en grandes séries et de la diversification des produits. Secteur par secteur, la croissance respective des firmes et de leurs marchés soulevait des problèmes nouveaux. Par exemple celui d'informer la clientèle visée sur la qualité des produits proposés. Dès la fin du $19^{\mathrm{e}}$ siècle, Michelin (1889) et le Chocolat Menier (1893) élargirent ainsi leurs débouchés en inaugurant la publicité de masse et de marque. Cependant que le droit des brevets entreprit de concilier l'intérêt du consommateur qu'il faut protéger contre les abus de pouvoir des monopoles avec l'intérêt du déposant soucieux de rentabiliser l'invention ${ }^{1}$.

Deux courants de pensée se sont détournés du modèle néo-classique et du postulat d'information parfaite. Le premier tire son origine de l'identification des fonctions du management. On doit ainsi à H. Fayol (1918) et à sa longue expérience d'industriel d'avoir distingué cinq fonctions élémentaires coiffées d'une fonction « administrative » relevant de la responsabilité du dirigeant suprême. Dès lors, le bien marchand de la théorie économique s'efface derrière les spécialités indispensables à sa production. La division du travail conduit l'entreprise à prendre des

1. La modernisation de ce droit fait suite aux précédents historiques qui remontent à Venise 1450, aux États-Unis (1790, 1794), la France $(1791,1844)$. 
décisions dont la complexité, la cohérence, la complémentarité instantanée et intertemporelle commandent les résultats. En tant qu'une organisation qui émet, filtre et reçoit des informations, la firme s'analyse aussi comme une suite de rendez-vous internes et de concert avec ses partenaires; des concertations cruciales pour sa compétitivité. L'analyse managériale ne peut rester indifférente au second courant de pensée qui traite de l'information en général et de ses limites à la suite de Hayek (1937, 1945), Simon et March (1958), Akerlof (1970) et Stiglitz (2002). Leurs travaux ont renouvelé l'analyse du fonctionnement des marchés et des rapports inter-entreprises en plaçant cette fois au premier plan le rôle de l'information, ses coûts, ses imperfections et les asymétries qui en résultent.

Ces deux tendances ouvrent la voie à la prise en compte du rôle stratégique, tout à la fois central et spécifique de l'information pour la conduite des entreprises. Un rôle central : à partir des années 1980, nombre de grands groupes se sont, à la suite de Toyota, considérés comme des systèmes d'information englobant sélectivement employés, clients, fournisseurs, sous-traitants, partenaires publics et associatifs et concurrents. Ils ont trouvé dans les TIC les moyens de s'aventurer dans la grande exportation, de délocaliser les chaînes de valeur, d'externaliser des activités non stratégiques, d'élargir leur clientèle d'abonnés, bref de mondialiser leurs activités.

Un rôle spécifique : l'information possède des propriétés singulières qui en font une ressource différente de ce qu'ont représenté autrefois le blé, l'acier et qu'assure aujourd'hui le pétrole. Ces ressources sont physiquement extérieures à leur utilisateur.
L'information agit en revanche de l'intérieur. Elle pénètre les esprits, imprègne les relations économiques, structure les pouvoirs, irrigue la société, nourrit les tensions sociales (Bienaymé, 2009).

Détenir une information peut conférer un avantage décisif pour l'action : l'utilité de l'information (I) pour le décideur dépend de l'état de ses connaissances antérieures (C) et du parti qu'il entend en retirer dans l'action qu'il envisage de décider (D). Trois moments clés précèdent en effet l'action, ce que nous illustrons par le triptyque Connaissances $\leftarrow \rightarrow$ Information $\rightarrow$ Décision ou effet C-I-D. Quand toute information ponctuelle reçue, qu'elle soit inattendue ou recherchée, est mise en rapport avec notre connaissance explicite et implicite, ce savoir hérité du passé lui donne son sens. L'information confirme, complète, infléchit ou contredit la connaissance que nous pensions détenir. Si elle est aberrante, extravagante, elle est rejetée la plupart du temps comme absurde. Si elle étonne modérément, elle retient l'attention.

L'utilité de l'information pour l'entreprise comme pour tout décideur varie en fonction de la décision qu'elle éclaire, des probabilités de succès de l'action qui s'ensuit. Plusieurs qualités confèrent de la valeur à une information donnée ; des qualités en partie inconciliables. Nous relevons les suivantes : exactitude, précision, comparabilité des données dans le temps et dans l'espace, crédibilité des sources, fraîcheur des données, pertinence, exclusivité. Les quatre premières citées concernent l'apport d'une information à la connaissance ; les trois dernières commandent directement l'action du récepteur.

Comme toute matière première, l'information livrée en vrac doit être si possible 
vérifiable et exploitable. Or, paradoxalement, nul décideur n'est assuré de la qualité de toutes les informations qu'il reçoit. Il ignore les conditions dans lesquelles certaines ont été émises et lui ont été transmises; les manipulations malintentionnées sont fréquentes. Premier paradoxe, l'information sur la qualité de l'information est inégale, asymétrique.

L'essor d'Internet ${ }^{2}$ s'explique par la baisse drastique des coûts de communication qui intervient au moment où les économistes attribuent un coût et une valeur à l'information, deuxième paradoxe. Cet événement de portée planétaire transforme les pratiques observées dans la conduite des entreprises. Faut-il en conclure qu'il est nécessaire de réviser une nouvelle fois la conception de la firme ou, à tout le moins, se bornera-t-on à signaler l'apparition de nouveaux types d'entreprises, comme le suggère la mode des business models et l'essor des platesformes électroniques?

Le numérique immerge les décideurs dans un océan d'informations à flux tendus. Ce phénomène inédit trouble la chaîne traditionnelle C-I-D. L'information $\mathrm{X}$ reçue au temps $\mathrm{T}$ remonte mal en amont vers le stock $\mathrm{C}$ de connaissances accumulées, car l'information pertinente se distingue mal d'une foule hétéroclite de messages souvent éphémères. Leur qualité est délicate à vérifier ; le temps disponible et l'attention, cette ressource rare, manque pour en juger (Simon, 1971 ; Citton, 2014). Comme le dit D. Cardon : « Lorsque l'offre d'information abonde, c'est désormais l'attention des publics qui constitue un bien rare et convoité » (p. 93). Le tri des données reçues à chaque seconde se fait mal. Et quand la décision d'acheter ou de vendre un titre financier est confiée à un algorithme, le lien entre l'information récente et la signification qu'on pourrait lui donner après réflexion disparaît. Par conséquent, le rapport de l'information à la connaissance se brouille. Le flux incessant d'informations nouvelles ou indéfiniment répétées relègue dans l'oubli les connaissances passées. Et le fait de confier l'archivage des données à des ordinateurs autorise indûment l'individu à se dispenser de l'effort de mémorisation. Or, l'atrophie de la mémoire personnelle est un danger pour l'esprit. En privilégiant les données de l'instant, on oublie l'avenir. Le « papillonnage cognitif » disperse l'attention et contribue à mettre les informations sur le même plan, quelle qu'en soit l'importance (Carr, 2011 ; Vargas Llosa, 2012).

L'information utile pour l'action en aval laisse entière la question classique de savoir s'il faut la garder secrète pour surprendre les concurrents, ou au contraire la diffuser pour attirer les partenaires auxquels on souhaite s'associer. Elle se pose avec d'autant plus d'acuité que les TIC valorisent les interactions personnelles, multiplient les occasions de coopérer. La réponse dépend en partie des données que les dirigeants considèrent comme «stratégiques » (Deville de Perrière, 2015). L'économie numérique déplace le champ des droits de propriété intellectuelle sans en supprimer l'intérêt, pas plus qu'elle n'ôte de son intérêt au secret des affaires.

On invoque habituellement l'accélération des événements préludant aux décisions pour qualifier de révolution l'essor de l'économie numérique. The Economist (2015b) relativise ce point de vue en niant

2. 3 milliards d'abonnés dans le monde, 26,2 millions en France avec une croissance de 3,7 \% l'an. 
qu'il y ait quelque chose de réellement nouveau au royaume des entreprises. L'avalanche d'informations adressées aux dirigeants donne l'illusion que l'accélération du tempo change l'entreprise dans son essence. Bien au contraire, selon The Economist, les TIC ne changeraient rien au fait constamment vérifié que l'entreprise, quelle qu'elle soit, transforme, à l'image des banques, les ressources du présent dans les produits à venir connus ou inconnus aujourd'hui.

Révolution ou simple illusion ? Pour laisser le lecteur en juger, on exposera dans ce qui suit ce que suggèrent en tendance, les pratiques managériales d'apparition récente. Le numérique a ses fans, mais le propos est moins de s'extasier sur l'outil et ses tentacules ${ }^{3}$ que d'observer l'usage qui en est fait. Si le secteur numérique représente un peu plus que $5 \%$ du PIB en France, il diffuse ses effets dans l'ensemble de l'activité économique et compterait pour un tiers de la croissance (modeste) du PIB. Le numérique s'impose par deux caractéristiques dominantes : l'omniprésence de l'information, la démesure du quantifiable. Ce qui se répercute sur la vitalité de l'appareil de production.

\section{II - L'OMNIPRÉSENCE DE L'INFORMATION}

Analysons la portée de cette révolution en évoquant les changements que l'omniprésence des TIC suscite dans la vie des affaires. On en retiendra de quatre sortes.

1) Dans la relation du producteur au client, le vendeur apprécie de plus en plus finement les besoins et les dispositions à payer des consommateurs. Les pays riches du Nord connaissent des problèmes spécifiques de débouchés pour des biens disponibles en abondance. En recueillant méthodiquement l'information sur le contenu des paniers, les distributeurs ajustent leurs commandes pour réduire leurs stocks. La profusion et la variété des produits créent un besoin d'information qui incite tout à la fois les clients à élargir leur éventail de choix et les vendeurs à cibler leurs clientèles. En s'appuyant sur la recherche d'informations par les clients potentiels, les annonceurs adaptent leurs messages et les producteurs diversifient leurs offres, fixent leurs prix et conditions de vente au plus près du consentement à payer de leurs clients. $\mathrm{Ce}$ qui revient à confisquer une grande partie de la rente de l'acheteur, même si en apparence ses vœux semblent satisfaits; il est vrai que cette pratique trouve sa justification dans la volonté de faire payer à chaque individu le vrai coût de la prestation fournie et d'éviter que certains clients ne soient avantagés aux dépens des autres.

Face à une demande volatile et de plus en plus informée, le vendeurs tentent de fidéliser la clientèle en offrant des conditions financières attirantes. On franchit un degré de plus avec les objets connectés dont le prestataire de services contrôle l'usage (GPS, irrigation agricole, santé, domotique, etc.). Ce procédé étend les perspectives concernant les abonnements des ménages et l'entretien des matériels.

Mais les pratiques promotionnelles ont leur coût. Les discriminations tarifaires pratiquées de longue date, notamment dans les transports, brouillent la vision que le client

3. Puces, satellites, fibre optique, capteurs, senseurs, écrans, logiciels, données, supports, cloud, applications, robots, drones, etc. 
se fait du prix à payer dans les ventes sous conditions. L'argument de la gratuité que sert Internet pour ses services trouble aussi cette vision. Grâce à la gratuité de leurs services, Google, Amazon, Facebook, Apple (GAFA) développent des réseaux d'abonnés dont la taille permet de se financer en vendant à des entreprises intéressées les informations qu'elles détiennent. Ces firmes ont un double marché, celui des internautes, celui des annonceurs: l'internaute de base devient à son insu le produit intermédiaire des agences de publicité qui le démarchent. Nous voilà bien loin des temps où la théorie prétendait voir dans la concurrence le moyen d'aligner le prix des biens sur leurs vrais coûts de production et où l'on pouvait tabler sur un prix d'équilibre du marché unique pour tous.

Les techniques de communication deviennent particulièrement subversives quand elles s'en prennent au monopole du droit de la propriété individuelle. En effet, les TIC apportent un concours décisif aux succès des sites de partage de biens durables, notamment dans les transports, les bureaux, les outillages, l'habitation, etc. La logique du droit d'accès à l'emploi de ces biens dicte des contrats distincts de ceux qui consacrent leur pleine propriété. Ce type d'échange procure des avantages substantiels : économie des coûts de transaction, gestion optimale du parc des biens concernés. Ainsi ce système remédierait à la sous-utilisation des voitures particulières dans des proportions considérables ${ }^{4}$.

Et l'arrivée annoncée de la voiture autonome et partageable rajeunira le parc collectif tout en réduisant l'activité des constructeurs, des compagnies d'assurances et des hôpitaux. Il reste à régler quelques questions concernant la fiabilité des services rendus, les obligations et la sécurité des contractants (Gille et Marchandise, 2015). La révolution en cours nous éloigne ainsi de l'économie de marché et des transactions à bout de bras entre acteurs indépendants (arms length competition) décrites dans les manuels d'économie.

2) Information et communication dans l'entreprise ont des exigences qui influent sur les relations employeurs/employés, et d'abord sur les habitudes de travail. Une influence non dépourvue d'ambiguïté. Les théories du management soulignent le fait que les TIC rendent possibles et souhaitables le partage des informations, la coopération horizontale et multilatérale des personnels à l'intérieur de l'entreprise. Mais la boulimie d'informations qui en résulte génère une bureaucratie indésirable : prolifération des contrôles et des reportings, complexification des processus de décision, incertitude des acteurs sur leur propre environnement et sur la portée du droit à l'erreur (Dupuy, 2015).

Les TIC autorisent les entreprises à diversifier leurs méthodes de recrutement par rapport aux procédés traditionnels : petites annonces des journaux, relations sociales et associations d'anciens diplômés, agences de travail intérimaire, pôles emploi publics. Le recrutement par la voie des réseaux sociaux permet aux partenaires potentiels d'apprécier plus finement les aspirations des uns et les compétences recherchées par les autres et d'organiser des jeux d'apprentissage pour accompagner les premiers pas des nouvelles recrues. Ces méthodes se développent d'abord dans les métiers issus du

4. De l'ordre de $96 \%$ (The Economist, 2015a). Il en est de même pour les bateaux de plaisance. 
numérique : programmeurs, logiciels d'applications, jeux vidéo, matériels informatiques et de télécoms...

Dans la mesure où l'emploi des TIC nécessite peu de capitaux, elles favorisent l'éclosion de formes d'activité plus individuelles telles que l'auto-entrepreneuriat ou encore le contrat de travail à la demande par lequel Amazon par exemple propose de payer ses livreurs à la tâche. Certains observateurs en concluent peut-être un peu vite au déclin du salariat.

3) Les TIC rendent les process de production de plus en plus flexibles. Les robots de la première génération associent l'électronique à la mécanique ; l'ingénierie moléculaire associée à l'informatique facilite l'essor des productions sur mesure. Ainsi, la fabrication additive exploite avec l'aide des ordinateurs et d'imprimantes 3D les propriétés chimiques de la lumière et des matériaux pour déboucher sur un vaste ensemble d'applications industrielles et d'objets à hautes performances (Aveni, 2015 ; The Economist, 2015c) ${ }^{5}$. Cette révolution n'en est qu'à ses débuts. Il faut aussi évaluer en comparaison des biens traditionnels la nouveauté des objets connectés et des fonctionnalités dont ils seront dotés : services préventifs, proactifs, à distance. On trouve d'ores et déjà plus de différences entre un tracteur connecté et un tracteur mécanique qu'entre celui-ci et l'animal de trait. Et comme le disent Escande et Cassini : "Les maîtres du logiciel comme Tesla, Google, Netflix, Uber se lancent dans des métiers qui ne sont pas les leurs. » (automobiles, films de série, thermostats, taxis).
4) Le concept de concurrence longtemps assimilé à une simple structure de marché connaît un nouvel avatar. La concurrence que nous avions définie comme «la recherche active d'une positon relative meilleure (par rapport à des acteurs stratégiquement indépendants) ou la lutte constante contre tout ce qui tend à la détériorer » (Bienaymé, 1976), subsiste avec ce que ces rivalités impliquent en termes de fusionsacquisitions. Mais à l'heure du numérique et de la floraison de réseaux interactifs, les occasions de coopérer se multiplient sans pour autant nécessiter de transferts de capitaux (Bienaymé, 1998). La concurrence entre marques s'accommode d'un recours à des plateformes communes. L'économie numérique fait alors basculer la concurrence d'une compétition entre acteurs indépendants vers une rivalité entre éco-systèmes (Porter et Heppelmann, 2014, 2015) composés de clubs de firmes qui sont à la fois partenaires, fournisseurs, clients sur certains composants (Apple/Samsung) et concurrents sur le produit fini, ce qui ajoute aux difficultés d'appréhender les pratiques anti- concurrentielles.

L'omniprésence de l'information rend-elle transparente pour autant la firme du monde digital ? Baigne-t-elle dans un environnement transparent ? En s'éloignant du paradigme de la firme selon Coase, l'entreprise contemporaine se rapprocherait-elle de l'entreprise néo-classique ? Certainement pas, même si la grande taille ne confère plus un avantage aussi décisif, l'entreprise émergente diffère de la firme de concurrence pure et parfaite sur deux points. D'abord, la transparence suppose que

5. Parmi les applications industrielles de la fabrication additive en gestation, citons Carbon 3D, Multi-jet fusion, Bioprinting, 4D Printing (avec molécules à mémoire régénérant les produits usagés). 
l'information circule sans effort particulier. Or, l'information stratégique se traque, se trie, se traite en fonction d'un objectif de rentabilité. L'entreprise du monde numérique ne se contente pas d'équilibrer ses recettes et ses coûts.; il lui faut des bénéfices pour durer. Ensuite, l'appétit d'information croît avec les moyens de la produire et de la diffuser ; une information aussi abondante fait prendre conscience de la complexité du monde et des difficultés d'interprétation que rencontre le décideur avant d'agir. Si les TIC facilitent la mondialisation des chaînes de valeur, l'essor du commerce international des tâches, elles exposent les groupes industriels et financiers à un environnement infiniment plus risqué que la plupart des TPE à clientèle locale sans pour autant épargner à celles-ci les joies de la bureaucratie électronique.

L'omniprésence de l'information se constate : on ne peut cependant se contenter de cette évidence. Les procédés utilisés pour traduire les réalités en données numériques, puis les exploiter et les propager participent à la démesure de nombreuses initiatives marquant la société contemporaine. Une démesure dont il convient de mettre en lumière certains aspects.

\section{III - LA DÉMESURE DU QUANTIFIABLE}

Si l'on perçoit les avantages de la révolution technologique en cours, il reste que le bon dosage du recours aux TIC pose question. Car ces techniques encouragent une tendance que l'on définira comme la démesure $\mathrm{du}$ quantifiable. Tentons d'abord d'en cerner la nature avant d'en explorer les répercussions sur l'organisation industrielle.
1) Ce type de démesure s'affirme à partir de la systématisation de la collecte d'informations sur des ensembles variés : entreprises, secteurs, régions, professions ou toute autre entité socio-politique. Les opérateurs, notamment des spécialistes tels que le quatuor GAFA ou des instituts de sondages, entendent exploiter à leur avantage ces mines d'informations que sont les mégadonnées. La concentration de grands volumes de statistiques sert à identifier, sans catégorisation a priori, l'existence de corrélations et de tendances qui sont débusquées grâce aux capacités de calcul des ordinateurs (Cardon, 2015). Ce procédé concerne les personnes, les objets, le grand public, les territoires, la société.

Il permet, par exemple, «d'enrichir la relation client ». La numérisation des données aborde l'être humain sous toutes ses facettes mesurables, appréhende ses besoins et ses habitudes dans leur infinie variété. Il n'en demeure pas moins que l'individu quantifié comme client n'est que la caricature de la personne. Certes, dans un domaine tel que la santé, les méga-données font progresser l'hygiène et les soins médicaux. Mais derrière le patient connecté, outre les dangers de l'automédication propres aux consultations en ligne, on oublie parfois que la malade souffre aussi de sa solitude. La communication sans contact humain reste abstraite, incomplète. En ce qui concerne les activités scientifiques et de R\&D, l'accumulation des corrélations est sans aucun doute féconde ; toutefois, elle ne dispense pas de rechercher les causalités (Porter et Heppelmann, 2014, 2015 ; Coupland, 2015), ni les idées au-delà des chiffres.

La conversion des signaux analogiques en un langage numérique rigoureux augmente 
la faculté d'appréhender dans les moindres détails les informations contenues dans les objets ou associées à leur emploi. En 2015, le « compteur communicant» Linky qu'ERDF a présenté sous son meilleur jour, comme outil d'aide aux économies d'énergie, livrera des informations dont l'usage peut être détourné à des fins indésirables.

2) Plus profondément, si la quantification progresse avec le numérique, la marchandisation progresse à son tour avec le quantifiable. Ainsi en est-il des fonctionnalités indispensables, secondaires, comme des menus services que les vendeurs intègrent dans leurs produits. Ces pratiques créent l'illusion répandue que n'importe quel service peut s'acheter (Hénaff, 2002 ; Sandel, 2012). De sorte que les TIC participent à cet impérialisme semble-t-il irrépressible du marché. Il est permis de penser, au nom d'un éthique de vérité et de justice, que la société doit préserver la part de l'humain qui relève du « hors de prix », la part de l'innumérisable.

3) La progression à l'infini du quantifiable influe sur les méthodes d'administration des sociétés. Les directions d'entreprises deviennent plus exigeantes sur les objectifs, les résultats et mettent en place des méthodes d'évaluation des personnels parfois difficilement tolérées (Dupuy, 2015). Les autoroutes de la communication ouvrent un appétit sans limites et propice aux abus : empiétement sur les temps de loisir, normes de qualité pointilleuses et imposées, procédures de contrôle, " taylorisme digital » (The Economist, 2015a). Paradoxalement, le numérique ne rapproche pas spontanément les acteurs qui sont les cadres opérationnels locaux, les concepteurs de produits et les bureaucraties intermédiaires animées par des fonctionnels experts en organisation informatique. Par les facilités de communication qu'il offre, le numérique envahit l'emploi du temps des personnels dirigeants particulièrement engagés dans les aventures de la mondialisation.

\section{IV - RÉPERCUSSIONS DE LA TRANSITION NUMÉRIQUE SUR L'APPAREIL DE PRODUCTION}

Microsoft, Google, Microsoft, Apple, Amazon et quelques autres façonnent le monde à venir en ce début de siècle. Ils doivent leur puissance digitale et leur prééminence à l'architecture de leurs systèmes et aux algorithmes de leur choix. Ils protègent leurs secrets de fabrication, notamment en pratiquant la course en tête des innovations. Ainsi, troisième paradoxe, l'avènement du numérique ne dissipe pas les asymétries d'information régnant sur ces marchés. À leur suite, l'appareil productif des économies développées évolue dans plusieurs directions aux effets contrastés.

La France qui s'est engagée timidement dans la transition numérique dépend toujours de l'étranger pour l'importation des matériels électroniques. Quoique tardif, son engagement est toutefois devenu manifeste en 2015 grâce à l'expansion du nombre de start-up qui font abondamment usage des TIC animées qu'elles sont par une génération de digital natives, ces enfants du numérique séduits par la dynamique des réseaux sociaux. Ce sont souvent des concepteurs et des développeurs de logiciels. Qu'ils trouvent ou non leur inspiration dans la Silicon Valley, ils s'adressent à un large éventail d'activités : recrutement de talents, tourisme, architecture, bio-technologies, informatique, jeux et loisirs, crowdfunding, etc. (Google). Ces jeunes pousses 
communiquent leurs projets à des apporteurs de capitaux (par exemple BPI, Orange, Vinci en France). Leurs perspectives de bénéfices sont certes fragiles. La dynamique des recettes (publicité, abonnements) et des coûts (économies d'échelle de réseau et d'adoption) laisse planer une incertitude sur le montant des profits à venir et leur localisation. L'économie d'Internet surestime la valeur de certaines start-up habiles à embellir leurs perspectives d'avenir. Mais il en est de même pour toute entreprises de création récente ${ }^{6}$.

Le secteur des assurances trouve à cet égard une occasion de s'impliquer dans le financement de ces nouveaux risques. Ainsi GAN Assurance s'est associée à Auchan, à l'Ordre des experts comptables et au journal Les Échos pour animer la plate-forme de financement participatif Lendopolis de prêts aux TPE. Le même assureur s'est associé au « Réseau Entreprendre », un club d'entreprises établies qui se charge d'accompagner de leurs conseils et de divers concours financiers les start-up de leur choix ${ }^{7}$. Ces apports en capitaux sont encore insuffisants pour faire accéder les meilleures d'entre elles à la taille critique internationale.

Les transactions directes d'entreprises à particuliers progressent fortement depuis 2010. Avec Amazon en tête, le commerce en ligne a atteint en France où l'on compte 34 millions de cyber-acheteurs, $57 \mathrm{M} €$ de chiffre d'affaires selon la commission des finances du Sénat (septembre 2015). Le site e-commerce Builders dresse un palmarès des performances des sites de distribution à l'usage du public anglophone.

Les gestionnaires de plates-formes numériques organisent aussi des transactions directes de particulier à particulier; ces sites collaboratifs sont des sortes de néomarchés portant sur des biens et des services variés (transports, logements, cours en ligne, etc.). La France découvre en 2015 sous le nom «d'uberisation » ce que l'on baptisa en 1998 au MIT. sous le néologisme « E-Lance Economy », désignant par là un type d'activité combinant la disponibilité des individus et leurs offres de compétences : l'offre des indépendants répond à la demande dans des délais courts (Malone et Laubacher, 1998). Citons ici les découvreurs : «We're in the dawn of the e-lance economy », (Thomas Malone). «E-lancers are electronically connected freelancers. They work together on project teams for a day, a week, a month, or longer - and then disperse and recombine to work on other projects » (Mieszkovski, 1999).

Ce foisonnement d'initiatives portera ses fruits en termes d'emplois; mais à plus court terme il fragilise une partie de l'appareil productif. Des professions d'intermédiation sont menacées par une concurrence d'un type nouveau. Par la disparition de nombreuses petites entreprises, ce sont des villages et des campagnes qui sont guettés par la désertification à moins qu'on n'ajuste la politique du numérique aux impératifs de l'aménagement du territoire.

6. Selon la Coface (mars 2015) le taux de défaillance des start-up était de 2,34 \% contre 2,54\% pour l'ensemble de la France.

7. Sous l'enseigne «Viva Technologys » Publicis et le groupe Les Échos ont créé une association qui a été rejointe par AXA, BNP Paribas, Orange, Google France et Ernst \& Young France. Son but est de multiplier les partenariats entre start-up et grandes entreprises. Elle organise à cette fin la réunion de 5000 start-up à Paris du 30 juin au 2 juillet 2016. 


\section{La transition numérique tend à fracturer le tissu industriel}

Sont vulnérable les PME traditionnelles et plus largement les entreprises installées dont les personnels habitués à l'ancien modèle hiérarchique vivent difficilement les changements touchant «leur» travail. Peut-on se prononcer sur les conséquences en découlant sur le nombre et la qualification des emplois ? Jusqu'à présent notre pays a importé ses robots et ses gros équipements électroniques en provenance des États-Unis, du Japon et de l'Allemagne. Les gains de productivité obtenus avec ces machines intelligentes et celles qui sont en gestation pourraient détruire durablement plus d'emplois qu'ils ne permettront d'en créer. Après avoir fait disparaître nombre d'emplois routiniers et peu qualifiés, la transition numérique, plus précisément l'intelligence artificielle, menace à son tour les emplois qualifiés et routiniers qui prédominent dans la population active, par exemple dans la banque, la presse, l'édition musicale, la distribution commerciale, les agences d'assurance, de voyage et de publicité, etc.

\section{L'organisation industrielle qui s'installe est vulnérable à divers égards}

La dynamique du glaive et du bouclier perturbe les entreprises exposées au risque de captation et de manipulation des données. Le numérique ne fait pas disparaître la notion d'information stratégique, sensible, qu'il faut apprendre à protéger. Sans tomber pour autant dans la paranoïa, les entreprises sont invitées à prendre en charge leur propre sécurité informatique. Les produits modernes sont des systèmes complexes exposés au risque de défaillance du moindre de leurs éléments; le trafic aérien, les centrales nucléaires ne sont pas complètement à l'abri des agressions d'où qu'elles viennent. Enfin, l'excès de confiance dans les algorithmes utilisés par les traders pour leurs opérations financières contribue en partie au dérèglement des marchés financiers. Le numérique ne remplace pas le discernement dont les décideurs doivent faire preuve.

On peut certes douter de la portée de la révolution Internet en comparaison de l'apport du tout à l'égout, de l'éclairage électrique, du moteur à combustion et du téléphone. On peut sous-estimer les innovations de la fin du $19^{\mathrm{e}}$ siècle et exagérer celles du présent (Gordon, 2013 ; Wolf, 2015). Mais il nous semble que la nature de la firme et des rapports qu'elle entretient avec les marchés subit de profondes transformations. Ni Coase, ni Walras ne résument dans leurs schémas la nature de la firme contemporaine. Tout en se rapprochant par certains côtés du modèle d'économie de marché du paradigme néoclassique, l'économie numérique s'en éloigne à d'autres égards. Elle s'en rapproche en flattant l'individualisme ambiant, en faisant fi des hiérarchies, en facilitant les conduites opportunistes et l'optimisation à court terme des choix, en privilégiant le contact multilatéral, hors intermédiaires professionnels. Elle s'en éloigne par le rôle déterminant conféré aux technologies de l'information et de la communication, par leur hypertrophie. Les prix à la consommation ne sont plus guère ce signal renseignant objectivement l'ensemble des acteurs du marché sur le coût des biens ; ce sont des instruments manipulés aux fins d'extraire la rente de l'acheteur à la plus petite unité de temps près; d'où la tentation de celui-ci 
d'échapper à cette emprise par le recours aux services en ligne. Le monde digital favorise l'apparition de techno-monopoles géants et de «marchés où le gagnant remporte toutes les mises » (Wolf, 2015). Les réseaux de partenariat associant petits producteurs et grands groupes relativisent le classement des entreprises par ordre de taille. Enfin, le numérique invite à revoir nos conceptions sur les rapports entre connaissance, information, décision et action. $\mathrm{Ce}$ que le numérique apporte en termes de confort matériel dans les opérations de production, dans les prestations de services crée de nouvelles obligations à l'entreprise. Son équipement intellectuel est soumis à de nouvelles exigences dans le domaine de la formation et des rapports interpersonnels.

\section{BIBLIOGRAPHIE}

Akerlof G. (1970). "The market for lemons: quality uncertainty and the market mechanism", Quarterly Journal of Economics, août, vol. 84, n⿳ 3, p. 488-485.

Aveni R. (d') (2015). “The 3D Printing Revolution”, Harvard Business Review, mai.

Bienaymé A. (1976). « Propos sur la concurrence », Revue française de gestion, $\mathrm{n}^{\circ}$ 3, jan-fév., p. $82-85$

Bienaymé A. (1998). Principes de concurrence, Economica, 470 p.

Bienaymé A. (2009). «Le pouvoir subversif de l'information », Commentaire, printemps, $\mathrm{n}^{\mathrm{o}} 125$, p. 37-46.

Cardon D. (2015). À quoi rêvent les algorithmes : nos vies à l'heure des big data, Éditions du Seuil, Paris.

Carr N. (2011). Internet rend-il bête ? Réapprendre à lire et à penser dans un monde fragmenté, Robert Laffont.

Citton Y. (sous la dir., 2014). L'économie de l'attention : Nouvel horizon du capitalisme?, Éditions La Découverte, Paris.

Coase R. (1937). “The Nature of the Firm”, Economica, vol. 4, n 16, novembre, p. 386-405.

Coupland D. (2015). «Sommes-nous autre chose que des données? », Problèmes Économiques, $1^{\circ}$ octobre, $\mathrm{n}^{\mathrm{o}} 3115$, p. 35-42.

Deville de Perrière D. (2015). «TIC, internet, réseaux sociaux : les nouvelles menaces », Problèmes Économiques, $1^{\mathrm{e}}$ juin, $\mathrm{n}^{\mathrm{o}} 3113$, p. 20-27.

Dupuy F. (2015). Lost in management : la vie quotidienne des entreprises au XXI siècle, Seuil, 284 p.

Escande P. et Cassini S. (2015). Bienvenue dans le capitalisme 3.0, Albin Michel.

Fayol H. (1918). Administration industrielle et générale, Dunod.

Gille L. et Marchandise J.-F. (2015). « Les enjeux de la régulation numérique », Problèmes Économiques, $\mathrm{n}^{\mathrm{o}} 3109$, avril 1, p. 23-33.

Google (2015). Annuaire des Start-up. 
Gordon R. (2013). "Is U. S. Economic growth over? Faltering innovation confronts the six headwinds", NBER Working Papers Series, $\mathrm{n}^{\mathrm{o}} 18315$.

Hayek F. (1937). "Economics and knowledge", Economica, n ${ }^{\circ}$ IV 33, février, p. 33-56.

Hayek F. (1945). "The use of knowledge in society", American Economic Review, XXXV, septembre, p. 519-530.

Hénaff M. (2002). Le prix de la vérité, Éditions du Seuil, Paris.

Knight F. (1921). Risk, Uncertainty and Profit.

Malone T. et Laubacher R. (1998). "The Dawn of E-Lance Economy”, Harvard Business Review, sep.-oct.

Marshall A. (1890). Principles of Economics.

Mieszkovski K. (1999). "New fast company" site New Fast Company, 31 octobre.

Porter M. et Heppelmann J. (2014). "How smart-connected products are transforming competition", Harvard Business Review, novembre.

Porter M. et Heppelmann J. (2015). "How smart-connected products are transforming companies", Harvard Business Review, octobre.

Sandel M. (2012). What money can't buy: the moral limits of markets, édition française Le Seuil, Paris.

Simon, H.A. (1971). "Designing organizations for an information-rich world", Computers, Communication, and the Public Interest, The Johns Hopkins Press, Baltimore, M. Greenberger, MD.

Simon H. et March J. (1958). Orgnaizations, Wiley.

Stiglitz J. (2002). "Information and the change in the paradigm in economics", American Economic Review, juin.

The Economist (2015a). "Digital taylorism", 12 septembre.

The Economist (2015b). "The Creed of Speed", 5 décembre.

The Economist (2015c). "New Materials for Manufacturing”, 5 décembre.

Vargas Llosa M. (2012). La Civilisation du Spectacle, Gallimard, Paris.

Wolf M. (2015). «Pourquoi les techno-opttimistes ont tort», Problèmes Économiques, $\mathrm{n}^{\mathrm{o}} 3115$, sept. I, p. 26-35. 